\title{
Desarrollo de una plataforma IoT para la gestión del riego de precisión
}

\author{
Puig Pérez-Barquero, Francisco ${ }^{1}$, Rodríguez Díaz, Juan Antonio², González Perea, Rafael 3, Camacho Poyato, Emi- \\ lio $^{4}$
}

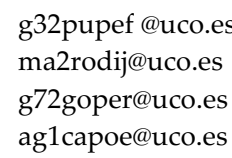

Resumen: Hoy en día el riego de precisión es cada vez más importante debido a la necesidad de optimizar el uso de los recursos agua y la energía, los cuales son cada vez más limitantes. Sin embargo, aplicar un riego de precisión no es una tarea fácil para la mayoría de los agricultores, ya que es necesario conocer los factores que influyen en el crecimiento del cultivo. El avance tecnológico está permitiendo que cada vez sea más factible medir, recopilar datos y analizar el manejo del riego, utilizando diferentes tipos de sensores y servidores, de esta manera se pueden calcular de forma automática las necesidades de riego y aplicar el agua de forma precisa justo cuando es más necesaria. No obstante, la mayoría de las plataformas IoT son sistemas cerrados que no permiten la integración con plataformas y sensores de terceros y se limitan a mostrar los valores de estos sensores de manera gráfica, sin entrar en el cálculo de las necesidades del cultivo y, por tanto, no son del todo útiles para funcionar como sistemas de apoyo a la toma de decisiones. El presente trabajo pretende mostrar la metodología para el desarrollo de una plataforma IoT de bajo coste para el riego de precisión, así como la integración de diferentes estrategias de riego en la misma. Para desarrollar la plataforma se ha utilizado el framework Django, de código abierto. Con el fin de conectar diferentes sensores a la plataforma se ha diseñado un dispositivo de toma de datos, de código abierto y eficiente energéticamente, que soporta una gran cantidad de protocolos de comunicación. Finalmente se ha calculado una programación de riego para una finca de estudio. Dicha finca consta de 44 hectáreas de almendros en intensivo y organizados en dos sectores de riego. Las estrategias se han desarrollado con el lenguaje de programación Python y se conectan automáticamente a la red de estaciones meteorológicas de la AEMET y a la página web de elTiempo.es para obtener tanto datos climáticos de la serie histórica como las predicciones para los próximos días. Todos estos datos se muestran en la plataforma de forma gráfica, a través de la cual también se pueden modificar las variables relacionadas con el sistema hidráulico, el cultivo y la explotación

Palabras clave: Riego de precisión, Cultivo del Almendro, TIC's, Software, Aplicación Web 


\title{
XXXVIII Congreso Nacional de Riegos
} CARTAGENA 2021

\section{Developing an IoT platform for smart irrigation management}

\author{
Puig Pérez-Barquero, Franciscoํㅜ, Rodríguez Díaz, Juan Antonio², González Perea, Rafael ${ }^{3}$, Camacho Poyato, Emi- \\ lio $^{4}$
}

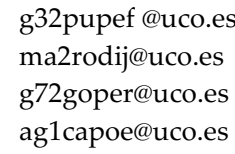

\begin{abstract}
Nowadays the smart irrigation is becoming increasingly important due to the need to optimise the use of water and energy resources, which are becoming more and more limiting. However, applying smart irrigation is not an easy task for most farmers, as it is necessary to know the factors that influence crop growth. Technological advances are making it increasingly feasible to measure, collect data and analyse irrigation management, using different types of sensors and servers, so that irrigation needs can be calculated automatically, and water can be applied precisely when it is most needed. However, most IoT platforms are closed systems that do not allow integration with third-party platforms and sensors and are limited to displaying the values of these sensors graphically, without entering the calculation of crop needs, and therefore are not entirely useful to work as decision support systems. This paper aims to show the methodology for the development of a low-cost IoT platform for smart irrigation, as well as the integration of different irrigation strategies in it. The open-source Django framework has been used to develop the platform. In order to connect different sensors to the platform, an open source, energy-efficient data acquisition device has been designed, which supports a large number of communication protocols. Finally, an irrigation schedule has been calculated for a study farm. This farm consists of 44 hectares of intensive almond trees organised in two irrigation sectors. The strategies have been developed with the Python programming language and are automatically connected to the AEMET weather station network and to the elTiempo.es website to obtain both weather data from the historical series and forecasts for the coming days. All this data is displayed graphically on the platform, through which the variables related to the hydraulic system, the crop and the farm can also be modified.
\end{abstract}

Keywords: Smart Irrigation, Almond trees, TIC's, Software, Web Platform 


\section{Introducción}

En España, al igual que ocurre en el resto de los países de la cuenca mediterránea, el agua es un recurso escaso, frágil e irregularmente distribuido. A la escasez de precipitaciones, la elevada evapotranspiración, la alta variabilidad espacial y temporal de las lluvias, la desigual distribución de los recursos hídricos y la frecuencia de las sequias, se unen problemas como son el aumento de la presión sobre los recursos hídricos, el déficit creciente que sufren algunas cuencas y la salinización o contaminación de acuíferos [1].

La agricultura de regadío es el principal consumidor de agua, suponiendo un $82 \%$ del total del agua consumida, según estimaciones del Instituto Nacional de Estadística (INE) en 2018. Por ello, en la actualidad, y más aún en el futuro, la agricultura de regadío se llevará a cabo bajo un escenario de escasez de agua. El suministro insuficiente de agua para el riego serä la norma más que la excepción, y la gestión del riego pasará de enfatizar la producción por unidad de área, hacia la maximización de la producción por unidad de agua consumida (Fereres \& Soriano, 2007). Para el cálculo de las estrategias de riego de precisión es necesario conocer los parámetros que afectan a la distribución de agua en el suelo y la planta. Sin embargo, conocer estos parámetros y calcular el balance de agua en el suelo no es una tarea fácil para la mayoría de los agricultores.

El desarrollo tecnológico, que implica la mejora y la disminución del coste de los sensores, supone un cambio importante en la agricultura. El IoT (Internet of Things) surge como la opción natural para las aplicaciones de gestión inteligente del agua [2]. Con el uso de esta tecnología se pueden desarrollar estrategias de riego de precisión, de manera autónoma, que permitan un mayor control de los diferentes factores que afectan al riego y mejoren la gestión de agua y energía en la explotación. Esto reduce costes, mejora la aplicación del riego y disminuye el impacto medioambiental, siendo cada vez más imprescindible su uso en la agricultura. Sin embargo, la mayoría de las plataformas y sensores comerciales son sistemas cerrados, que no permiten la integración con sensores y plataformas de terceros, lo que limita en gran medida su potencial.

El presente trabajo pretende mostrar la metodología que se ha seguido para el desarrollo de un sistema IoT, de bajo coste y de código abierto, para la automatización de las estrategias de riego de precisión en una finca comercial de almendros. Este sistema permite la integración con diferentes plataformas IoT y soporta una gran cantidad de sensores comerciales.

\section{Materiales y métodos (Trabajo de innovación: descripción de la innovación)}

\section{1. Área de estudio}

El proyecto se ha llevado a cabo en una finca de almendros situada en Córdoba, España. El clima en esta zona se caracteriza por inviernos suaves, con temperaturas que rondan los $10^{\circ} \mathrm{C}$ y veranos calurosos, con temperaturas medias superiores a los $27^{\circ} \mathrm{C}$. Las precipitaciones anuales rondan los $592 \mathrm{~mm}$, pudiendo llegar algunos años a superar los $1.000 \mathrm{~mm}$. El terreno presenta una textura que varía entre franco-arcillosa y franco-arenosa, en función de la zona.

Dicha finca consta de dos variedades de almendro, Soleta y Lauranne, distribuidas en dos parcelas con una superficie productiva de 22 y 21 hectáreas respectivamente. La plantación es de tipo intensiva con un marco de 6 × 3,5 metros. El riego es por goteo, con un caudal de gotero de 1,2 L/h,_y está dividido en dos sectores, uno para cada variedad de almendro, y cada uno consta de dos bombas. Una de las bombas se encargará de llevar el agua del río Guadalquivir hasta un depósito de un millón de litros. La segunda bomba es utilizada para aplicar riego a los distintos sectores. Ambas bombas se alimentan 
mediante energía fotovoltaica. La finca cuenta también con un sistema de fertirriego, de modo que le aplican los fertilizantes directamente en el agua de riego.

Las características del sistema de riego por sectores se representan en la Tabla 1. Los valores de uniformidad y eficiencia de riego se han obtenido de un ensayo de campo que realizó la Universidad de Córdoba

Tabla 1. Características del sistema de riego por sectores. Fuente: elaboración propia.

\begin{tabular}{ccccc}
\hline Sector & No Goteros & $\begin{array}{c}\text { Caudal Gote- } \\
\text { ros (1/h) }\end{array}$ & $\begin{array}{c}\text { Uniformi- } \\
\text { dad del } \\
\text { riego (\%) }\end{array}$ & $\begin{array}{c}\text { Eficiencia del riego } \\
\text { (\%) }\end{array}$ \\
\hline 1 & 87000 & 1.18 & 95.5 & 90 \\
2 & 93000 & 1.15 & 90.4 & 90 \\
\hline
\end{tabular}

\subsection{Materiales}

\subsubsection{Dispositivos IoT}

Para permitir la conexión de los sensores con la plataforma IoT se han desarrollado unos dispositivos de toma de datos, compatibles con diferentes sistemas de comunicación y protocolos de salida. Estos dispositivos se caracterizan por:

- Amplio rango de instalación: Los dispositivos han sido desarrollado con el objetivo de soportar un gran rango de sistemas de comunicación, ya que se suelen instalar en lugares remotos, en los que muchas veces no hay conexión a internet. Estos sistemas de comunicación son: GSM, Sigfox, LoraWAN, NB-IoT, Bluetooht y Wi-Fi. En este trabajo se ha optado por utilizar la tecnología SigFox.

- Eficientes energéticamente: Debido a la dificultad para acceder a los dispositivos, estos deben ser autosuficientes energéticamente. Estos tienen una batería de Li-Ion recargable de $2600 \mathrm{mAh}$ y 3.7V que se recarga a través de un panel solar de $250 \mathrm{~mA}$.

- Compatibles con una gran variedad de sensores: Para conseguir medir una mayor cantidad de parámetros que afectan a el riego, es necesario que los dispositivos sean compatibles con la gran mayoría de sensores comerciales. Entre los protocolos que soporta se encuentran: SDI-12 (Serial Digital Interface), RS-485, I2C (Inter-Integrated Circuit) y SPI (Serial Peripheral Interface). Los dispositivos también son compatibles con sensores analógicos.

Estos dispositivos se han programado con el software Arduino y envía los valores tomados por los sensores cada $15 \mathrm{~min}$ a la plataforma IoT. 


\subsubsection{Sensores de suelo}

El cálculo de la humedad en la zona radicular se ha realizado mediante dos sensores (Figura 1), que miden la humedad de suelo, la conductividad eléctrica y la temperatura, instalados a 30 y $45 \mathrm{~cm}$.

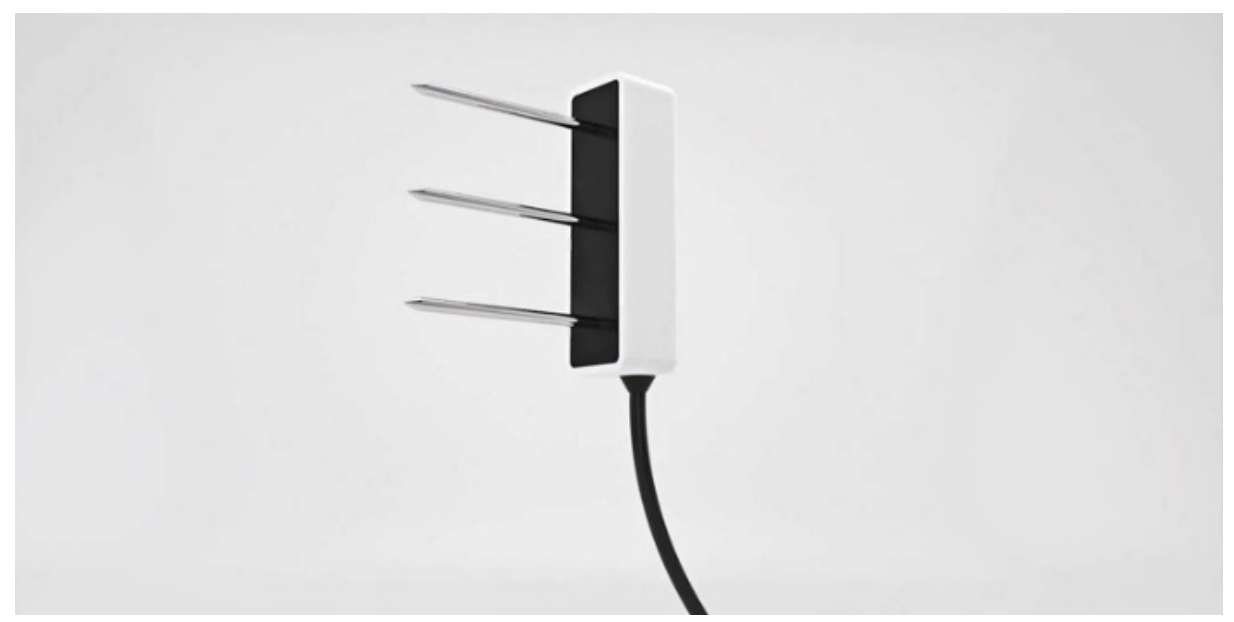

Figura 1. Sonda de humedad Teros 12.

Estos sensores se comunican con el dispositivo de toma de datos mediante el protocolo de comunicación SDI-12.

\subsubsection{Servidor}

Se ha utilizado la Raspberry Pi 3 Model B+ como servidor (Figura 2). La Raspberry es un ordenador de placa reducida, de bajo coste y consumo reducido, lo que lo hace ideal para utilizarlo como servidor para proyectos en desarrollo.

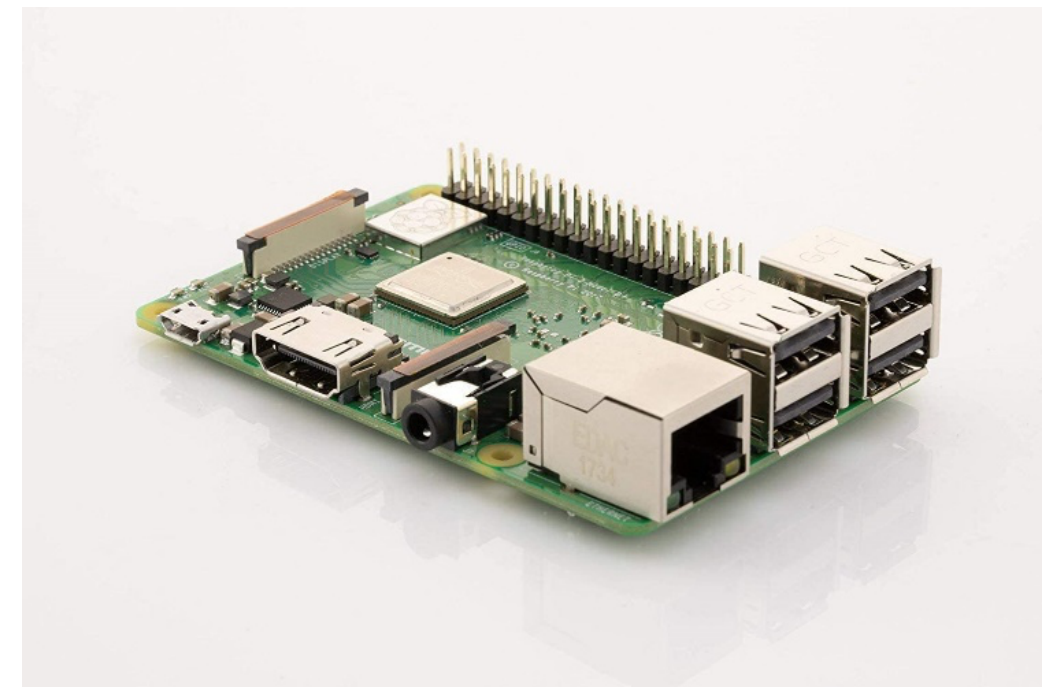

Figura 2. Raspberry Pi 3 Model B+ 


\section{Congreso Nacional de Riegos CARTAGENA 2021}

\subsection{Metodología}

La metodología se puede dividir en dos partes, el desarrollo del sistema IoT y el cálculo de la programación de riego.

La arquitectura del sistema IoT (Figura 3) se puede dividir en tres capas:

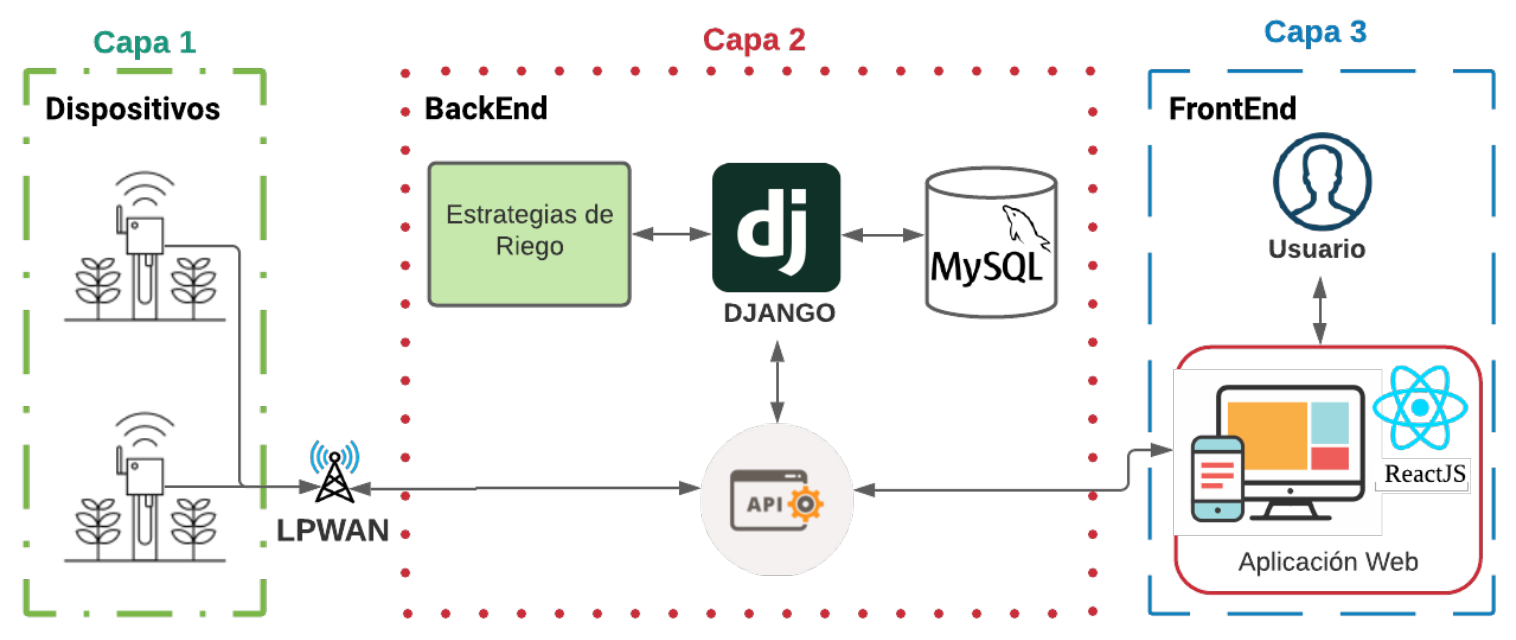

Figura 3. Arquitectura del sistema IoT. Fuente: elaboración propia.

La capa 1 representa los dispositivos de toma de datos descritos en el apartado anterior. Estos dispositivos se conectan con la API REST (Application Programming Interface. REpresentational State Transfer) del backend a través de los sistemas de comunicación LPWAN.

La capa 2 representa el backend de la plataforma. Se ha utilizado el framework de Django (https://docs.djangoproject.com/), de desarrollo web y de código abierto, escrito en Python, que sigue un patrón de diseño conocido como Modelo-Vista-Controlador (MVC). En esta capa se encuentran el algoritmo de riego de precisión, desarrollado en el lenguaje de programación Python, en el que se calculan las diferentes estrategias de riego. Para comunicar las tres capas, se ha desarrollado una API REST utilizando la herramienta "Django Rest Framework".

La última capa es el frontend que contiene la interfaz gráfica de la aplicación e interactúa con la segunda capa a través de su API REST. Para su desarrollo se ha utilizado la librería ReactJS.

Todo el sistema se ha desarrollado de manera que se puede desplegar tanto en la nube como insitu, en cualquier miniordenador. 


\subsubsection{Programación de riego}

En la aplicación se puede escoger entre dos tipos de estrategias: 1- Reponer al 100\% las necesidades de agua, 2- Riego deficitario controlado (RDC). La Figura 4 muestra un esquema del cálculo de la programación del riego en función de la estrategia escogida

Para la programación del riego de precisión se ha utilizado la metodología propuesta por la Organización de las Naciones Unidas para la Agricultura y la Alimentación (FAO) [3].

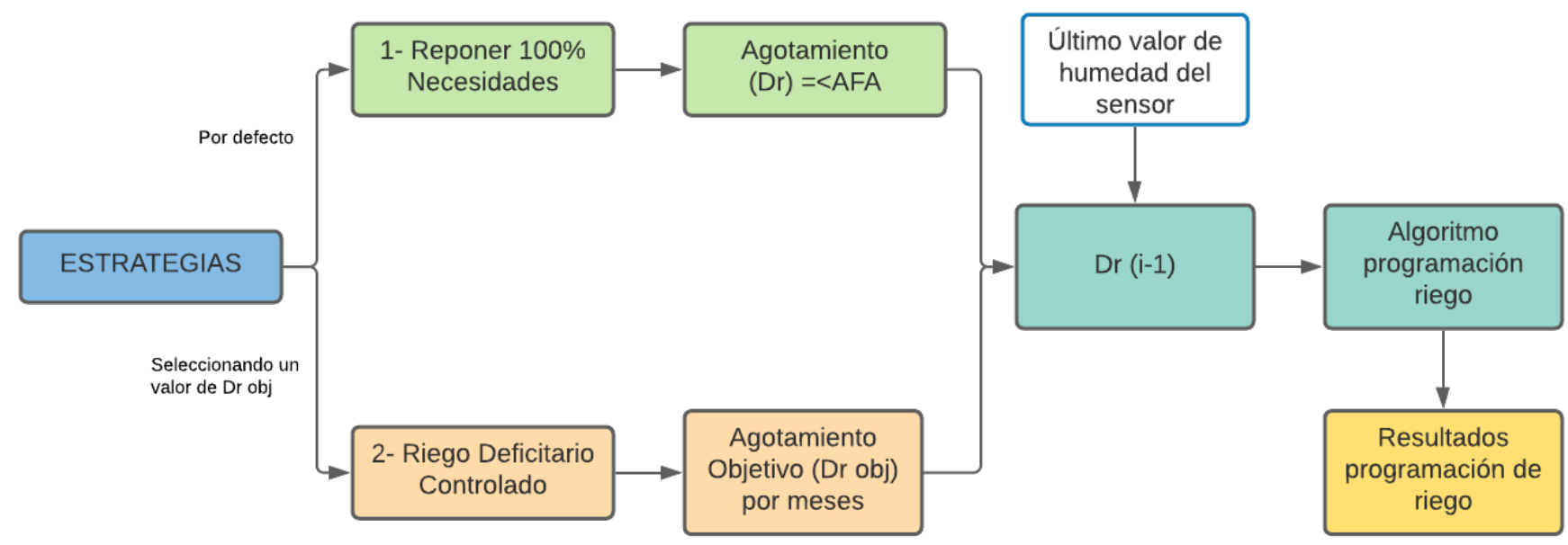

Figura 4. Esquema de la programación del riego para las diferentes estrategias. Fuente: elaboración propia

Como se observa en el esquema de la figura anterior, la programación de riego por defecto utiliza la estrategia de reponer $100 \%$ las necesidades hídricas y calcula el tiempo de riego que el sistema debe estar funcionando, para que el agotamiento de humedad en el suelo sea igual o menor al Agua Fácilmente Aprovechable (AFA). Este proceso lo repite para cada día de los próximos siete. De esta forma, aplicando el algoritmo utilizado para la programación de riego, que se explicará en el siguiente apartado, se obtiene la lámina bruta y el tiempo de riego necesarios para que el suelo no presente agotamiento de humedad.

Si se quisiera realizar un RDC se asigna un valor de agotamiento objetivo que establece el nivel máximo de agotamiento que el suelo debe presentar. De esta manera se puede reducir los aportes hídricos en aquellos periodos fenológicos en los que aplicar un déficit hídrico controlado no afecte sensiblemente a la producción y a la calidad de la cosecha, mientras que en el resto del ciclo del cultivo se garantice el $100 \%$ de las necesidades hídricas.

$D_{r, i-1}$ se calculará todos los días a partir de los valores que presenten los sensores de humedad instalados en la finca 


\section{Congreso Nacional de Riegos CARTAGENA 2021}

\subsubsection{Algoritmo Programación Riego}

El diseño del algoritmo de programación del riego va a seguir el esquema mostrado en la figura 5

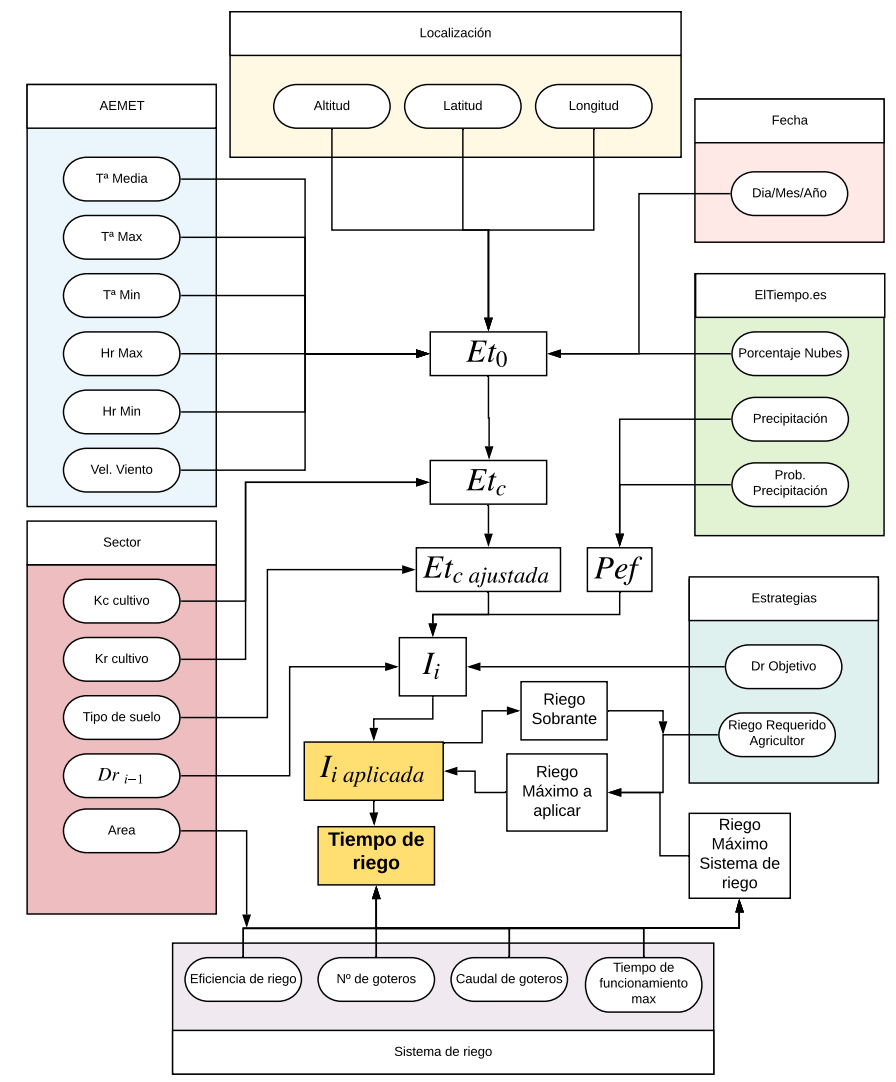

Figura 5. Esquema del diseño de la programación del riego. Fuente: elaboración propia

En primer lugar, se calcula la evapotranspiración de referencia $\left(E T_{0}\right)(\mathrm{mm})$ que depende únicamente de los valores climatológicos de la zona de estudio, los cuales se obtienen a través de la API de la AEMET OpenData

En segundo lugar, se calcula la evapotranspiración del cultivo $\left(E T_{c}\right)(\mathrm{mm})$ que utiliza el valor del coeficiente del cultivo $(\mathrm{Kc})$ y la $E T_{0}$ calculada anteriormente. El valor de $E T_{c}$ es menor cuando la cantidad de agua en el suelo está por debajo del Agua Disponible Total (ADT), ya que la transpiración del cultivo se reduce, por lo que debe ser ajustado en función del tipo de suelo, dando lugar a la evapotranspiración del cultivo ajustada $\left(E T_{\text {c ajustada }}\right)(\mathrm{mm})$.

Tras esto se realiza un balance de agua en el suelo para calcular la lámina neta de riego $\left(I_{i}\right)(\mathrm{mm})$, considerando las entradas (precipitación efectiva) y las salidas (evapotranspiración del cultivo.). Para el balance de agua es necesario el agotamiento del suelo del día anterior $\left(D r_{i-1}\right)(\mathrm{mm})$, que informa sobre la humedad del suelo, y el agotamiento del suelo al que se quiere llegar $\left(D r_{\text {objetivo }}\right)(\mathrm{mm})$.

Una vez calculada $I_{i}$, se calcula el tiempo que el sistema de riego debe de estar funcionando. Estas variables van a depender de las limitaciones del sistema hidráulico (Área, eficiencia del riego, n⿳o de goteros, caudal de goteros y tiempo de funcionamiento máximo) y de las limitaciones de agua que el agricultor especifique (riego requerido). Los parámetros referentes a la localización, sistema de riego y a las estrategias, se encuentran almacenados en la base de datos y pueden ser modificados desde la aplicación para ajustar la programación del riego a cualquier sector, cultivo y necesidades del agricultor 


\section{Congreso Nacional de Riegos CARTAGENA 2021}

\subsubsection{Despliegue}

Finalizado el diseño de la aplicación, es necesario pasar a la fase de producción (Figura 6). Esta fase consiste en desplegar la web en un servidor para que esté operativa las 24 horas y pueda ser accesible desde cualquier parte

Como ya se ha comentado, se ha optado por la Raspberry pi 3+ como servidor y se ha utilizado la herramienta Docker, que permite la automatización y el despliegue de la aplicación en diferentes dispositivos. La aplicación se ha desarrollado para ser lo más eficiente posible, permitiendo su funcionamiento en este "miniordenador". La elección de este servidor atiende a aspectos económicos.

\section{Resultados y discusión (Trabajo de innovación: resultados obtenidos en ensayo o en explotación comer-} cial)

El sistema IoT ha sido probado durante una campaña de riego completa. La aplicación web ha facilitado la visualización tanto de los valores de los sensores (Figura 7) como de las estrategias calculadas por el algoritmo de riego (Figura 8).

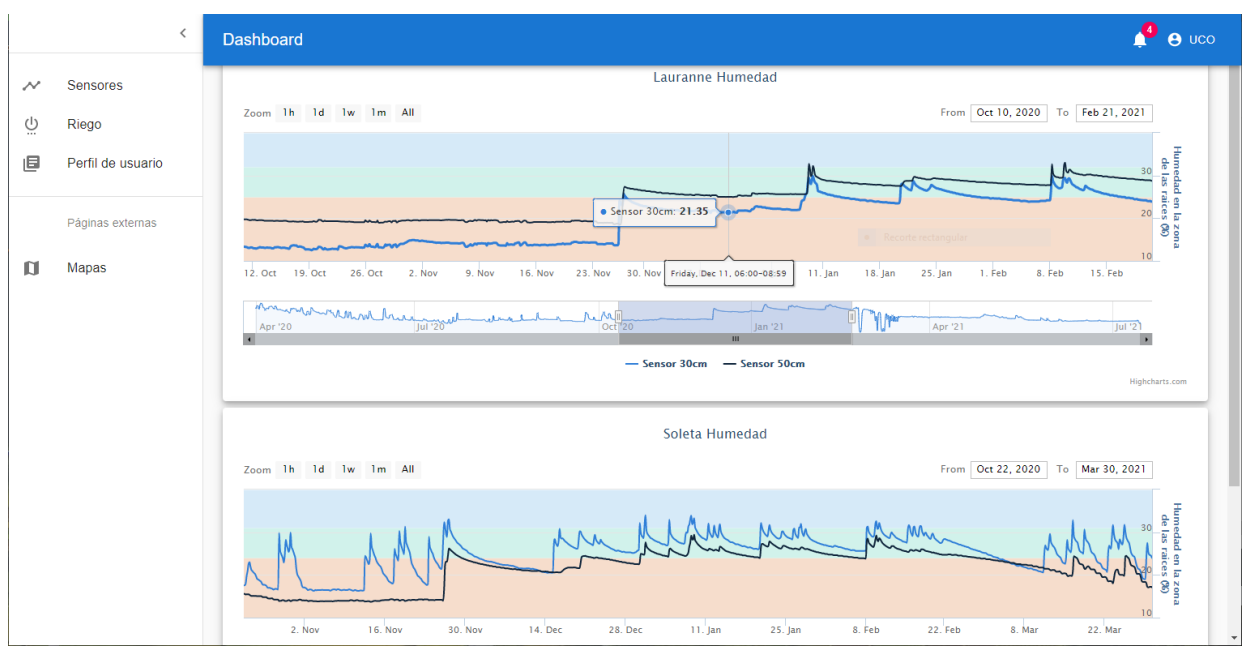

Figura 7. Página principal de la aplicación web. Fuente: elaboración propia

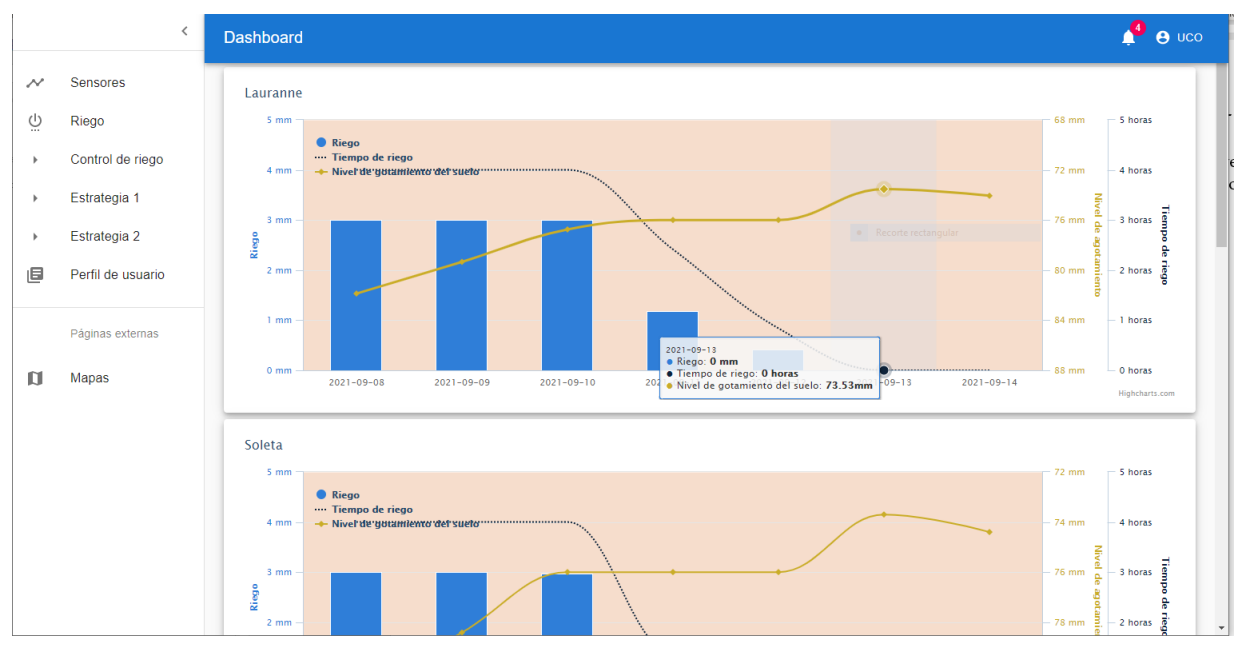

Figura 8. Página de estrategias de la aplicación web. Fuente: elaboración propia 


\section{Conclusiones}

En este trabajo se ha desarrollado un sistema IoT para controlar en tiempo real un riego de precisión, permitiendo una programación óptima, sencilla y personalizada para diferentes estrategias.

Se ha automatizado el cálculo de las necesidades de riego según la metodología de FAO. haciendo uso de las APIS de la AEMET y elTiempo.es, la aplicación realiza una predicción semanal de la variación de la humedad en el suelo.

La aplicación web implementa también el cálculo del tiempo que el sistema de riego debe funcionar a diario para aplicar el riego de precisión.

La aplicación ha sido desarrollada haciendo uso de dos de las herramientas más utilizadas hoy en día en desarrollo web (ReactJS y Django). Esto permite que dicho proyecto sea ampliamente escalable y útil de cara al mundo profesional.

Finalmente, la aplicación se ha pasado a modo producción y se ha instalado en un miniordenador, permitiendo así la instalación rápida, económica y segura en la explotación.

\section{Referencias}

1. García Morillo, J.; Rodríguez Díaz, J.; Montesinos, P., Hacia el riego de precisión en el cultivo de fresa en el entorno de Doñana, [Tesis doctoral]. 2015

2. Kamienski, C.; Soininen, J.P.; Taumberger, M; Dantas, R; Toscano, A.; Salmon Cinotti, T; Filev Maia, R.; Torre Neto, A, Smart Water Management Platform: IoT-Based Precision Irrigation for Agriculture, Sensors 2019, 19, 276.

3. Allen, R.G., Pereira, L.S., Raes, D., Smith, M. Crop evapotranspiration - guidelines for computing crop water requirements. FAO Irrigation and drainage paper 1998; 56. Food and Agriculture Organization, Rome.

4. Fereres, E., Soriano, M.A. Deficit irrigation for reducing agricultural water use. Journal of Experimental Botany 2007, Volume 58, Issue 2, 1, 147-159 